\title{
Semi-direct Gauge-Yukawa Mediation
}

\author{
Zhaofeng Kang, ${ }^{1}$ Tianjun Li, ${ }^{1,2}$ Tao Liu, ${ }^{1}$ and Jin Min Yang ${ }^{1}$ \\ ${ }^{1}$ Key Laboratory of Frontiers in Theoretical Physics, Institute of Theoretical Physics, \\ Chinese Academy of Sciences, Beijing 100190, P. R. China \\ ${ }^{2}$ George P. and Cynthia W. Mitchell Institute for Fundamental Physics, \\ Texas A\&M University, College Station, TX 77843, USA
}

(Dated: October 10, 2018)

\begin{abstract}
We propose semi-direct Gauge-Yukawa mediation of supersymmetry (SUSY) breaking. The messenger fields mediating SUSY breaking to the visible sector do not directly couple with the goldstino field, and instead they have gauge and Yukawa interactions with some primary messenger fields which couple directly with the goldstino fields. From the explicit Feynman diagram calculations for the SUSY breaking soft masses, we find that the SUSY particle spectra can be realistic. Especially, this generalization of semi-direct gauge mediation solves the massless gaugino mass problem since the holomorphic soft mass terms of the messenger fields can be generated by Yukawa couplings. We also provide some arguments that this scenario can be realized naturally in some dynamical SUSY-breaking models such as the ISS-like model.
\end{abstract}

PACS numbers: 12.60.Jv, 14.70.Pw, 95.35.+d 


\section{INTRODUCTION AND MOTIVATION}

The mechanism for supersymmetry (SUSY) breaking is an open topic. Due to the treelevel sum rule for the SUSY-breaking spectrum, namely the supertrace theorem, SUSY must be broken (spontaneously) in the hidden sector [1]. Then how to mediate the SUSY-breaking effects to the visible sector is a crucial issue. So far several schemes have been proposed, among which the Gauge Mediated SUSY Breaking (GMSB) is a quite promising approach because it can automatically avoid the notorious flavor problem [2] (for reviews, see, e.g., [3]). In particular, the direct gauge mediation has recently attracted much attention, in which the messengers play a role in determining the SUSY-breaking vacua [4]. However, this attractive approach easily suffers from some problems, for example, the suppressed gaugino mass problem [5], the Landau pole problem, and the fine-tuning problem. On the other hand, the indirect gauge mediation usually does not have the gaugino mass problem but it has a demerit of adding messengers by hand. A recent study on such messenger gauge mediation is given in Ref. [6].

No matter direct or indirect gauge mediation, the messenger fields $\Phi$ must carry the SUSY-breaking information in a form of the mass splittings $\left(m_{\phi, \pm}^{2}-M_{\phi}^{2} \neq 0\right)$ between the fermionic and bosonic components which are degenerate in a SUSY-preserved theory. But how the messengers obtain such mass splittings is unknown, and in the previous studies a singlet $X=\langle X\rangle+\theta^{2} F$ is simply introduced as the SUSY-breaking source which couples directly with the messengers. This is the so-called Minimal Gauge Mediation (MGM) and has been widely considered in phenomenology.

Note that such a simplification in MGM may hide some questions, for instance, why the messengers have to couple directly with $X$ to obtain mass splitting? In principle, the mass splitting can be achieved in a cascade way, i.e., some primary messengers $\phi_{0}$ (neutral under the Standard Model (SM) gauge group $G_{S M}$ ) mediate the SUSY-breaking to some secondary messengers $\phi_{1}$ via the hidden sector gauge interaction with a gauge group $G_{h}$ or Yukawa interaction, and this dynamical process can be successive if necessary, until finally mediate the SUSY-breaking to the visible sector. In this paper, we assume the simplest cascade patten, i.e., the two-step cascade mediation, as depicted in Fig. 1. In fact, such a two-step cascade mediation via pure gauge interaction is nothing but the semi-direct gauge mediation studied intensively in the literature [7-9]. However, the similar mediation mechanism with additional Yukawa interaction has not been studied, which will be examined in this article.

The conventional semi-direct gauge mediation suffers massless gaugino mass problem, since the secondary messengers does not acquire holomorphic soft terms [10, 11]. However, with Yukwa-assisted mediation, the holomorphic soft terms arise at one loop, thus gauginos can indeed become massive. We shall show that a calculable cascade SUSY-breaking with 
the minimal Kähler potential, based on a very general setup, is viable only when both gauge and Yukawa interactions are turned on. Namely, this hybrid cascade gauge-Yukawa mediation (HCGYM) can provide the viable SUSY particle spectra in some cases. The crucial point is the presence of non-vanishing supertrace in the messenger sector which links to the visible sector and modifies the sfermion masses drastically.

Note that our cascade scenario has one essential difference from the gauge mediation with messenger sector added by hand, i.e., our scenario can be naturally realized dynamically such as in the Intriligator-Seiberg-Shih (ISS)-like models [12]. In other words, the messengers in our scenario are a natural part in the hidden sector which breaks SUSY. We simply take a different way to gauge the so-called global flavor symmetry in the hidden sector. However, in our study we will start from a class of weakly coupled effective O'Raifeartaigh (OR) models, characterized by cubic terms and some large global symmetry $\supset G_{h} \times G_{S M}$ (some will be gauged properly), without any elaboration on their dynamical realization.

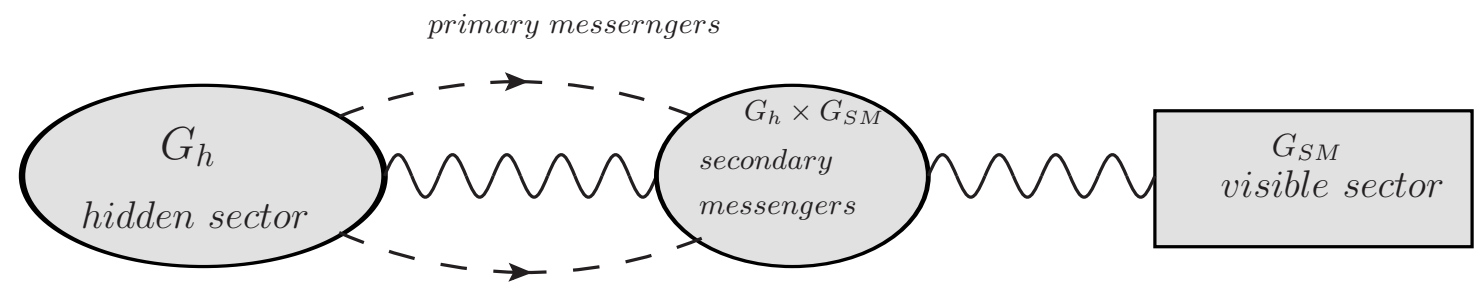

FIG. 1: A schematic diagram showing the structure of the two-step HCGYM.

The paper is organized as follows. In Section II we propose a general framework for HCGYM and then calculate the SUSY breaking soft terms via the secondary messengers. Our calculations will be performed explicitly in a very general setup. In Section III we analyze in what conditions the realistic soft masses in the visible sector can be achieved, and comment on the possible general features of the HCGYM. Finally, discussions and conclusion are given in Section IV.

\section{HYBRID CASCADE GAUGE-YUKAWA MEDIATION}

\section{A. The Primary SUSY-Breaking Mediation}

First we focus on SUSY breaking mediation without specifying any concrete model. Generally, a cascade mediation via gauge and Yukawa (hyprid) interactions has a typical structure as follows 
(i) A hidden sector, which breaks SUSY, is composed of Goldstino superfield $X$ and the primary vector-like messenger fields $\left(\phi_{i}, \bar{\phi}^{i}\right)$ with $i$ being the gauge/flavor index of the hidden sector group $G_{h}$ like $S U(N)$.

(ii) The secondary messenger fields $(F, \bar{F})$, which are charged under $G_{h}$ and the SM gauge group, and the vector-like fields $(f, \bar{f})$, which only carry the SM quantum numbers, both have supersymmetic mass terms and obtain SUSY-breaking effects via the interaction with the hidden gauge fields as well as from the cubic Yukawa coupling terms (discussed below).

(iii) Some cubic terms must be present, which couple one primary and one secondary messenger fields with the SM vector-like fields, or couple the two secondary messengers with one primary messenger. However, the cubic terms with two primary fields are not allowed by the SM gauge invariance. Note that if we turn off these cubic terms, the hidden sector SUSY-breaking will not be affected due to the fact that the secondary messengers are not relevant to the SUSY-breaking dynamics although they couple with the hidden sector. Then our scenario will just be reduced to the semi-direct gauge mediation discussed in Ref. [8].

We stress that our scenario is significantly different from the semi-direct gauge mediation [8]. The semi-direct gauge mediation [8] interpolates the MGM and the direct gauge mediation. It introduces a messenger sector that does not affect the SUSY-breaking and does not have to couple with $X$. However, it uses the messenger sector to probe the hidden sector only via some hidden gauge interactions. In our approach, the direct renormalizable couplings in the superpotential play a central role. As a result, the spectrum of the secondary messengers and also the visible fields is modified greatly. The presence of such cubic terms can arise in dynamical models, and, moreover, the mass scale can be dynamically determined. In other words, our framework regards the messenger sector as a built-in part of the dynamical model building, rather than introduced by hand.

With the above general setup, we propose to use the effective OR model which shows the dynamical structure. In our convention, we choose a basis in which the light messengers are diagonal while the hidden sector takes a general form

$$
\begin{aligned}
W= & {\left[\mathcal{F} X+\left(\lambda_{a b} X+m_{a b}\right) \bar{\phi}_{a}^{i} \phi_{b i}\right] } \\
& +\left(\lambda_{a} \phi_{a i} \bar{F}^{i} f+\bar{\lambda}_{a} \bar{\phi}_{a}^{i} F_{i} \bar{f}\right)+\left(M_{F} F_{i} \bar{F}^{i}+M_{f} f \bar{f}\right) .
\end{aligned}
$$

Here, the index $a$ does not refer to any symmetry and we require $M_{F, f} \ll \max \{\langle X\rangle, m\} \sim$ $M_{h}$, which is defined as the typical mass scale in the hidden sector, while $\sqrt{\mathcal{F}}$ determines the SUSY-breaking scale. Note that the multiple pairs of secondary messengers have additive 
contributions and thus do not change our conclusion. By the way, we may embed the SM gauge group into the group $G_{h}$ and then it induces the massless gaugino mass problem [5]

$$
M_{\lambda} \propto \frac{\partial}{\partial X} \log \operatorname{det} \mathcal{M}_{F}^{\text {primary }}=0
$$

where $\mathcal{M}_{F}^{\text {primary }}$ denotes the supersymmetric mass matrix for the hidden sector messenger.

Note that our general setup can be equipped with the $R$-symmetry. With the $R$-symmetry our framework can be considered as a generalization of the (extra)ordinary GMSB [13] with the cubic terms and the extra gauge group $G_{h}$. Then the particle charges under $R$-symmetry are

$$
\begin{aligned}
& R(X)=2, \quad R(F)+R(\bar{F})=2, \quad R(f)+R(\bar{f})=2, \\
& R\left(\bar{\phi}_{a}\right)+R\left(\phi_{b}\right)=0\left(\text { for } \lambda_{a b} \neq 0\right), \quad R\left(\bar{\phi}_{a}\right)+R\left(\phi_{b}\right)=2\left(\text { for } m_{a b} \neq 0\right), \\
& R\left(\phi_{a}\right)+R(\bar{F})+R(f)=2\left(\text { for } \lambda_{a} \neq 0\right), \quad R\left(\bar{\phi}_{a}\right)+R(F)+R(\bar{f})=2\left(\text { for } \bar{\lambda}_{a} \neq 0\right) .
\end{aligned}
$$

To make gauginos massive, $R$-symmetry must be broken. Here, we will not scrutinize the $R$-symmetry breaking mechanism. Instead, we simply assume it can be realized by $\langle X\rangle \neq 0$. This can be achieved by introducing some proper structures in the hidden sector, leading to the spontaneous breaking of the $R$-symmetry radiatively [14] or at the tree-level [5, 15].

Next, we discuss the SUSY-breaking encoded in the secondary messengers. They feel the SUSY-breaking effects through the following ways. One is through the conventional gauge mediation at two-loop level, of the non-holomorphic form such as $m_{F . g}^{2}|F|^{2}$ (Because $F$ and $f$ are similar, for simplicity, we will only focus on the former.). They also receive the direct one-loop contributions from the Yukawa couplings, which are calculated explicitly in Appendix IV A. Moreover, there are two-loop contributions, which are $\propto \lambda_{a}^{4}$ and can be extracted using the wave function renormalization method [16, 17]. In short, these three terms are given by

$$
\begin{aligned}
m_{F, g}^{2} & \simeq \frac{2 g_{h}^{4}}{\left(16 \pi^{2}\right)^{2}} \frac{\mathcal{F}^{2}}{M_{h}^{2}} \frac{N^{2}-1}{2 N}, \\
m_{F, Y 1}^{2} & =m_{F F^{*}}^{2}=i\left(\Sigma_{4}+\Sigma_{5}+\Sigma_{6}\right), \\
m_{F, Y 2}^{2} & =m_{F F^{*}}^{2}=\frac{\lambda_{a}^{4}}{\left(16 \pi^{2}\right)^{2}}(N+2) \frac{F^{2}}{M_{h}^{2}},
\end{aligned}
$$

where $g_{h}$ is the gauge coupling of $G_{h}$ and its value will be constrained by phenomenology. The explicit expressions of $\Sigma_{i}$ functions are given in Eqs. (37), (38), and (39). Such terms enter into the diagonal elements of the bosonic components of the secondary messengers, and behave as the $D$-term SUSY-breaking. In additional to the non-holomorphic terms, the other soft terms are holomorphic terms, namely $m_{F \bar{F}}^{2} F \bar{F}+$ h.c., which is generated at 
one-loop level due to the Yukawa interaction and proportional to the secondary messenger masses

$$
m_{h o l}^{2} \equiv m_{F \bar{F}}^{2}=m_{F^{*} \bar{F}^{*}}^{2}=i\left(\Sigma_{1}+\Sigma_{2}+\Sigma_{3}\right) \propto M_{F},
$$

where we have set $M_{F} \simeq m_{f}$. This term contributes to the non-diagonal mass terms of the secondary messengers and acts as the conventional $F$-term SUSY-breaking. It is absent in conventional semi-direct gauge mediation, rendering gaugino massless at the leading order of SUSY-breaking.

A comment is in order. For the SDGM there is a robust gaugino screen theorem [17]. It is derived through the renormalization of the real physical superfield

$$
R(\mu)=S(\mu)+S^{\dagger}(\mu)+\frac{T_{G}}{8 \pi^{2}} \log \operatorname{Re}(\mathrm{S}(\mu))-\sum_{r} \frac{T_{r}}{8 \pi^{2}} \log \mathcal{Z}_{r}(\mu),
$$

where $S(\mu)$ is the holomorphic gauge coupling that runs only at one-loop and $r$ runs over light fields at scale $\mu$. The screening depends heavily on the replacement $\mu_{F}^{2}=\frac{M_{F} M_{F}^{\dagger}}{\mathcal{Z}_{F}^{2}\left(\mu_{F}\right)}$ after across all messenger scales, leading to $R(\mu)$ independent on $X$ at leading order. However, just like the analysis in [21] which introduced chiral messengers so that the two chiralities of the messengers have different wave functions and thus invalidate the replacement, in our case the chiral Yukawa coupling between the secondary and primary messengers can also invalidate the replacement. Consequently, the screening is avoided.

By the way, in our framework we assume that $G_{h}$ is not Higgsed. If it is Higgsed, the gauge mediation has some modification and the modification extent is controlled by a new parameter $y=M_{V}^{2} / M_{h}^{2}$ with $M_{V}$ being the vector boson mass scale [18]. When it tends to be zero, we have the complete gauge group, while, oppositely, we have no gauge symmetry at the messenger scale. A sufficiently high $M_{V}$ is not a desired case and the reason will be discussed in the following.

\section{B. Gaugino and Sfermion Masses}

In the above we have studied the SUSY-breaking mediated to the secondary messengers by hybrid mediation. Now we calculate the SUSY-breaking mediated to the visible sector by the conventional GMSB. In contrast to the simple GMSB model where the hidden sector messenger spectrum has a vanishing supertrace, in our cascade framework the supertrace is nonzero due to the radiative corrections which are essentially from the holomorphic soft mass term $m_{F \bar{F}}^{2}$. In the basis $(F, \bar{F})$, the mass matrix of the scalar component is

$$
\mathcal{M}_{B}^{2}=\left(\begin{array}{cc}
M_{F}^{2}+D & F \\
F & M_{F}^{2}+D
\end{array}\right) \text {, }
$$


where $F=m_{\text {hol }}^{2}$ can be effectively treated as a spurion superfield $X$ which has a vanishing vacuum expectation value (VEV) for the lowest component but has a non-zero $\theta^{2}$ component. From this point of view, our framework provides a natural realization of multi-spurion fields, which will be helpful to implement general gauge mediation [19]. The term $D=m_{\text {nhol }}^{2}$ measures the supertrace. It has deep implication in the soft masses in the visible sector. The explicit calculations have been carried out in Ref. [20], and we use their results to calculate the soft masses of gauginos and sfermions.

In the limit of small SUSY-breaking $D, F \ll M_{F}^{2}$, the generic sfermion mass sqaure is approximately given by

$$
m_{\widetilde{f}}^{2} \simeq \sum_{a} C_{a}\left(\frac{\alpha_{a}}{4 \pi}\right)^{2}\left[2 \frac{F^{2}}{M_{F}^{2}}+D\left(-\frac{2}{3} \frac{F^{2}}{M_{F}^{4}}-4 \log \frac{\Lambda^{2}}{M_{F}^{2}}+4\right)\right],
$$

where $C_{a}$ is the Dykin index of $\tilde{f}$ related to the three SM gauge groups. $\Lambda$ is the ultraviolet cutoff scale related to a high scale at which $D$ and $F$ are generated, which, in fact, is the primary messenger scale $\Lambda \sim M_{h} \gg M_{F, f}$. This logarithmic UV-dependence is a character of the cascade gauge mediation. As for the gaugino masses, the $D$-type contribution does not affect their masses significantly, and thus they take a conventional form

$$
M_{\lambda_{a}} \simeq \frac{\alpha_{a}}{4 \pi} \frac{F}{M_{F}} .
$$

Now some comments are due regarding the property of the soft masses in the presence of $D$-type contribution. ¿From Eq. (10) we obtain

$$
m_{\tilde{f}}^{2} \simeq \sum_{a} C_{a}\left(\frac{\alpha_{a}}{4 \pi}\right)^{2}\left(2 \frac{F^{2}}{M_{F}^{2}}-4 D \log \frac{\Lambda^{2}}{M_{F}^{2}}\right) .
$$

So, depending on the sign of $D$, the sfermion mass square can be either enhanced $(D<0)$ or reduced $(D>0)$. As calculated in the previous section, there are several sources of nonholomorphic terms, and in the following we will show that a pure Yukawa cascade model is not viable practically.

First, in the cascade gauge mediation, a contribution from pure gauge interaction is necessary. In the limit $g_{h} \rightarrow 0$, generically the one-loop effect is dominant and scales as (the index $a$ will be dropped for simplicity)

$$
D=m_{F F^{*}}^{(1)} \sim \operatorname{sign}(\mathrm{D}) \times \frac{\lambda^{2}}{16 \pi^{2}} \frac{\mathcal{F}^{2}}{M_{h}^{2}},
$$

we will find that the sign is not predicted uniquely but dependent on the parameters in the hidden sector. On the other hand, the holomorphic term sales as

$$
F \sim \frac{\lambda^{2}}{16 \pi^{2}} \frac{\mathcal{F}}{M_{h}} \times M_{F},
$$


which is suppressed by the light supersymmetic mass term of the secondary messengers. Then we find that the $D$-type contribution dominates over the sfermion mass term. Concretely, in Eq. (10) the second term is about $\left(\log \frac{\Lambda}{M}\right) 64 \pi^{2} / \lambda^{2}$ times larger than the first term which is at the same order of gaugino mass. As a result, it leads to a splitting spectrum, which will incur large fine-tuning in the Minimal Supersymmetric Standard Model (MSSM).

The above conclusion is obtained only when the holomorphic and non-holomorphic SUSYbreaking terms are generated at the same loop level. We note that in some cases the two-loop contributions can dominate over the one-loop contributions which is suppressed by higher order of SUSY-breaking $\propto \mathcal{F}^{4} / M_{h}^{6}$ due to some subtle cancellation [20]. This happens when the hidden sector essentially contains only one mass scale $\propto \mathcal{F} / M_{h}$ which is irrelevant to the Yukawa couplings between $\Phi$ and $X$, as in the minimal GMSB with $X$ being the spurion field. In our model, this is approximately satisfied in the limit $\langle X\rangle \gg m$, but, unfortunately, a realistic spectrum cannot be obtained. In fact, the $D$-type contribution scales as

$$
D \sim \frac{1}{\left(16 \pi^{2}\right)^{2}} \lambda^{4} \frac{\mathcal{F}^{2}}{M_{h}^{2}} \text {. }
$$

Then the sfermion mass is given by

$$
m_{\text {sfermion }}^{2} \sim\left(\frac{\alpha_{a}}{4 \pi}\right)^{2} \frac{\lambda^{4}}{16 \pi^{2}} \frac{\mathcal{F}^{2}}{M_{h}^{2}}\left[2-4(N+2) \log \left(\frac{\Lambda^{2}}{M_{h}^{2}}\right)\right]
$$

which is always negative and unacceptable since it breaks the SM gauge group $G_{S M}$ at the messenger scale.

Now we consider the second limit $\lambda, \bar{\lambda} \rightarrow 0$ with a nonzero $g_{h}$. In this case we recover the conventional semi-direct gauge mediation scenario. The study in Ref. [10,11] pointed out that, independent of the details of the hidden sector, the doubly charged messengers do not acquire $F$-type SUSY-breaking information from it and thus the gaugino mass vanishes at the leading order (gaugino screening). In Ref. [21] some complicated chiral messener fields are proposed to overcome this difficulty. In our HCGYM approach we try to solve this by turning on the Yukawa couplings.

In our HCGYM scenario, we find that for a proper value of $g_{h} / \lambda$, the sfermion mass square can be reduced as a result of non-vanishing supertrace. The sfermion masses receive a $D$-type contribution as

$$
\operatorname{sign}(D) \frac{\lambda^{2}}{16 \pi^{2}} \frac{\mathcal{F}^{2}}{M_{h}^{2}}+\frac{2 g_{h}^{4}}{\left(16 \pi^{2}\right)^{2}} \frac{\mathcal{F}^{2}}{M_{h}^{2}} \frac{N^{2}-1}{2 N} .
$$

Therefore, for $\operatorname{sign}(D)=-1$, there exists cancellation between the gauge and Yukawa contributions. This $D$-type contributions approximately vanishes and the sparticle spectrum reduces to the MGM case when

$$
\frac{g_{h}}{4 \pi} \sim\left(\frac{\lambda}{4 \pi}\right)^{1 / 2}\left(\frac{N}{N^{2}-1}\right)^{1 / 4}
$$


In fact, we can tune the couplings to obtain our required soft masses. Note that the reduced sfermion spectrum generically requires a tuning of the ratio $\lambda / g_{h}$, which arises from the cancellation between the one-loop effect $\left(\propto \lambda^{2}\right)$ and the two-loop effect $\left(\propto g_{h}^{4}\right)$. By the way, increasing $N$ helps to lower the value of $g_{h}$, which, however, may induce the Landau pole for $S U(3)_{C}$ gauge coupling.

If the two-loop Yukawa corrections are dominant, different results will be obtained. Then the $D$-type contribution is

$$
\frac{1}{\left(16 \pi^{2}\right)^{2}} \lambda^{4} \frac{F^{2}}{M_{h}^{2}}+\frac{2 g_{h}^{4}}{\left(16 \pi^{2}\right)^{2}} \frac{\mathcal{F}^{2}}{M_{h}^{2}} \frac{N^{2}-1}{2 N} .
$$

Due to the fact that both $D$ - and $F$-type contributions come from the two-loop level, they have the same sign and the additional gauge contribution makes the sfermion mass quite large (negative).

Our above analysis is just a rough estimation. In the next subsection we will give a concrete example and perform the numerical calculations to show that our framework indeed works.

\section{A Concrete Example and A Preliminary Dynamical Realization}

To show that our HCGYM scenario can satisfy the required property, we now study numerically a concrete example. For this simple example the superpotential is assumed to be

$$
W=X \bar{\phi}_{1} \phi_{1}+\mu\left(\bar{\phi}_{1} \phi_{2}+\bar{\phi}_{2} \phi_{1}\right)+\lambda \phi_{2} F \bar{f}+\lambda \bar{\phi}_{2} \bar{F} f+m_{F} F \bar{F}+m_{f} f \bar{f},
$$

where $X$ is regarded as the spurion superfield, both $(\phi, \bar{\phi})$ and $(F, \bar{F})$ belong to the fundamental representation of the hidden gauge group $S U(N)$ while $(f, \bar{f})$ is neutral. We set $\langle X\rangle=M+F_{X} \theta^{2}$ with $M=1, \mu=1$ and $F_{X}=0.1$. Then we get the $D$ and $F$ terms as

$$
\begin{aligned}
& D=m_{F F^{*}}=-0.003 \times \frac{1}{16 \pi^{2}} \lambda^{2}+0.008 \times \frac{g_{h}^{4}}{\left(16 \pi^{2}\right)^{2}} \frac{N^{2}-1}{2 N}, \\
& F=m_{F \bar{F}}^{2}=0.07 \times \frac{1}{16 \pi^{2}} \lambda^{2} M_{F} .
\end{aligned}
$$

Another messenger pair $(f, \bar{f})$ also obtain soft $D$ - and $F$-type corrections which are enhanced by a group factor $N$, but receive no corresponding gauge contribution since they are charged under $G_{S M}$ only. With all these contributions, from Eq. (10) we find that for the sfermion masses the $D$-type contribution and $F$-type contribution has a ratio:

$$
R=-2(1+N) \log \frac{\Lambda^{2}}{M_{f}^{2}} \times \frac{\left(-16 \pi^{2} \lambda^{2}\right)+g_{h}^{4} \frac{N-1}{2 N} 0.008}{\left(0.07 \lambda^{2}\right)^{2}\left(N^{2}+1\right)} .
$$


Note that this result is independent of $M_{F}$. In Fig. 2 we plot this ratio as a function of $g_{h}$ (setting $\lambda=1$ ). As pointed previously, the ratio can be negative and thus can reduce the sfermion masses with some tuning of $\lambda / g_{h}$.

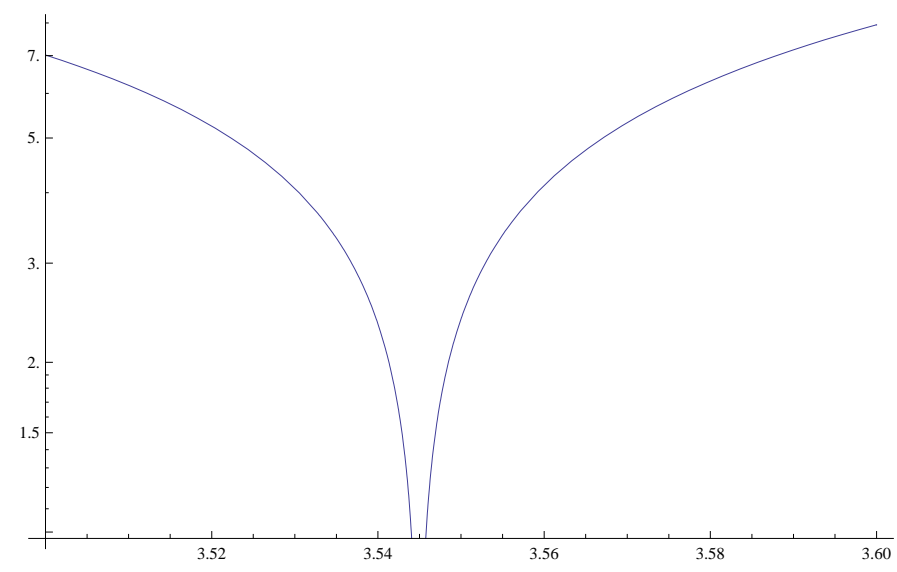

FIG. 2: . The ratio of $D$-type and $F$-type contributions $|R|$, as a function of $g_{h}$. Here we take $N=4$ and $\lambda=1, \Lambda / M_{F}=10^{4}$. The right-handed half curve covers the region with $R<0$.

Let us comment on the dynamical realization of our framework. In principle, any conventional semi-direct gauge mediation models can be updated to our HCGYM scenario by coupling the secondary messengers to the hidden sector. Another more natural model can be found in Ref. [22], which studied the metastable SUSY-breaking vacua in the $S U\left(N_{c}\right)$ supersymmetric QCD (SQCD) $(\mathcal{N}=1)$ in the superconformal window $3 / 2 N_{f}<N_{c}<2 N_{f}$ with $N_{f}$ being the number of the vector-like quarks. Based on the SQCD in Ref. [23], with some deformations, Ref. [22] studied its Seiberg dual description

$$
W=K p \widetilde{q}+L \widetilde{p} q+N q \widetilde{q}+M_{p} p \widetilde{p}-\mu^{2} N .
$$

The flavor symmetry is $S U\left(N_{f}^{(1)}\right) \times S U\left(N_{f}^{(2)}\right)$ and the magnetic gauge group is $S U(\widetilde{N})$ with $\widetilde{N} \equiv N_{f}^{(1)}+N_{f}^{(2)}-N_{c}$. Various fields are assigned in the representation as

\begin{tabular}{c||c|c|c} 
& $S U\left(N_{f}^{(1)}\right)$ & $S U\left(N_{f}^{(2)}\right)$ & $S U(\widetilde{N})$ \\
\hline$N$ & $\bar{N}_{f}^{(1)} \otimes N_{f}^{(1)}$ & 1 & 1 \\
\hline$q+\widetilde{q}$ & $\bar{N}_{f}^{(1)} \oplus N_{f}^{(1)}$ & 1 & $\widetilde{N} \oplus \widetilde{N}$ \\
$p+\widetilde{p}$ & 1 & $\bar{N}_{f}^{(2)} \oplus N_{f}^{(2)}$ & $\widetilde{N} \oplus \widetilde{\widetilde{N}}$ \\
\hline \hline$K+L$ & $\bar{N}_{f}^{(1)} \oplus N_{f}^{(1)}$ & $\bar{N}_{f}^{(2)} \oplus N_{f}^{(2)}$ & 1
\end{tabular}

Aside from the last line, this just gives the content of the ISS model [12]. It has a SUSY- 
breaking vacuum, with superfields parameterized as

$$
\begin{aligned}
& q=\left(\begin{array}{c}
\mu+\sigma_{1} \\
\phi_{1}
\end{array}\right), \quad \tilde{q}=\left(\begin{array}{ll}
\mu+\sigma_{2} & \phi_{2}
\end{array}\right), \quad N=\left(\begin{array}{cc}
\sigma_{3} & \phi_{3} \\
\phi_{4} & X
\end{array}\right), \\
& p=\phi_{5}, \quad \tilde{p}=\phi_{6}, \quad L=\left(\begin{array}{ll}
\phi_{7} & \tilde{Y}
\end{array}\right), \quad K=\left(\begin{array}{c}
\phi_{8} \\
Y
\end{array}\right) .
\end{aligned}
$$

Expanding around this vacuum, we obtain

$$
\begin{aligned}
W= & \left(X \phi_{1} \phi_{2}-\mu^{2} X+\mu\left(\phi_{1} \phi_{4}+\phi_{2} \phi_{3}\right)\right)+\mu\left(\phi_{5} \phi_{8}+\phi_{6} \phi_{7}\right) \\
& +\left(Y \phi_{2} \phi_{5}+\widetilde{Y} \phi_{1} \phi_{6}\right)+M_{p} \phi_{5} \phi_{6} .
\end{aligned}
$$

As shown in Ref. [22], when the flavor symmetry satisfies the relation

$$
N_{f}^{(2)}<2 \widetilde{N}<N_{f}^{(1)}+N_{f}^{(2)}<3 \widetilde{N},
$$

the theory is weakly coupled at IR and has tree-level SUSY-breaking vacua. Choosing $N_{f}^{(2)}=5, N_{f}^{(1)}=2, \tilde{N}=3$, and embedding $G_{S M} \subset S U\left(N_{f}^{(2)}\right)$, we find that $\phi_{1-4}$ play the role of primary messengers while $\phi_{5-8}$ are secondary messengers. From Eq. (27) it can be clearly seen that the hidden sector SUSY-breaking effect mediated by gauge and Yukawa interactions to the secondary messengers indeed appear in the model. Of course, it is not a realistic model since the $R$-symmetry is not broken. To make it realistic, further modifications in the hidden sector are required, which is beyond the scope of this paper.

\section{DISCUSSION AND CONCLUSION}

We proposed a pattern of cascade gauge-Yukawa mediation of SUSY-breaking based on an explicit Feynman diagram calculations for the soft mass terms. We found that the realistic soft masses for gauginos and sfermions can be obtained in the case that the conventional messenger fields obtain the SUSY-breaking effects through gauge and Yukawa interactions with the hidden sector. This hybrid scenario can easily avoid the massless gaugino mass problem and reduce the sfermion masses by some fine-tuning between $\lambda$ and $g_{h}$.

Finally, we note that very recently such a cascade patten was utilized for SUSY breaking (not mediation) in Refs. [24, 25]. And the $R$-symmetry spontaneous breaking at the twoloop level $[26,27]$ is also a result of cascade effect.

\section{Acknowledgments}

This work was supported by the National Natural Science Foundation of China under grant Nos. 10821504, 10725526 and 10635030, by the DOE grant DE-FG03-95-Er-40917, and by the Mitchell-Heep Chair in High Energy Physics. 


\section{APPENDIX}

\section{A. Soft Mass Terms from the One-Loop Yukawa Mediation}

Here we present the explicit one-loop calculations for the soft terms of the secondary messengers, using dimensional regularization. First of all, the mass square matrix of the primary messenger bosonic components $\left(\phi, \bar{\phi}^{*}\right)$ and the Dirac fermion mass matrix are respectively given by

$$
\begin{aligned}
& \mathcal{M}_{B}^{2}=\left(\begin{array}{cc}
(\lambda X+m)^{\dagger}(\lambda X+m) & \lambda \mathcal{F} \\
(\lambda \mathcal{F})^{\dagger} & (\lambda X+m)^{\dagger}(\lambda X+m)
\end{array}\right), \\
& \mathcal{M}_{F}=\lambda X+m
\end{aligned}
$$

where $\mathcal{M}_{F}$ is a $N_{p} \times N_{p}$ matrix while $\mathcal{M}_{B}^{2}$ is $2 N_{p} \times 2 N_{p}$. They can be diagnolized by the unitary matrix $U, N_{L}$ and $N_{R}$ respectively

$$
\begin{aligned}
U^{+} \mathcal{M}_{B}^{2} U & =M_{1}, \\
N_{L} \mathcal{M}_{F} N_{R} & =M_{2} .
\end{aligned}
$$

For the convenience of our following calculations, we parameterize the matrix in a block form

$$
U=\left(\begin{array}{cc}
A & B \\
C & D
\end{array}\right), \quad M_{1}=\left(\begin{array}{l}
m_{1} \\
m_{2}
\end{array}\right)
$$

Then the soft terms are given by

$$
V_{\text {soft }}=\left(m_{F \bar{F}}^{2} F \bar{F}+\text { h.c. }\right)+m_{F F^{*}}^{2} F F^{*}+m_{\bar{F} \bar{F}^{*}}^{2} \bar{F} \bar{F}^{*}
$$

The messenger fields $(F, \bar{F})$ get SUSY-breaking soft mass terms through one-loop diagrams

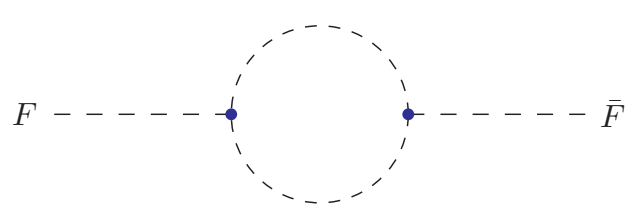

$(a)$

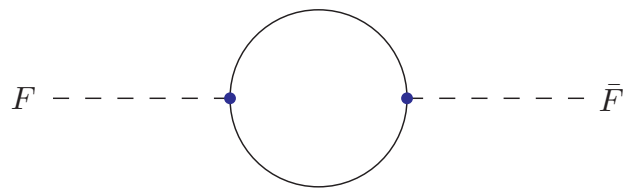

(b)

FIG. 3: Feynman diagrams contributing to the $F$-type soft terms.

shown in Fig. 3 and Fig. 4, which are given by

$$
\begin{gathered}
m_{F \bar{F}}^{2}=m_{F^{*} \bar{F}^{*}}^{2}=i\left(\Sigma_{1}+\Sigma_{2}+\Sigma_{3}\right), \\
m_{F F^{*}}^{2}=m_{\bar{F} \bar{F}^{*}}^{2}=i\left(\Sigma_{4}+\Sigma_{5}+\Sigma_{6}\right),
\end{gathered}
$$




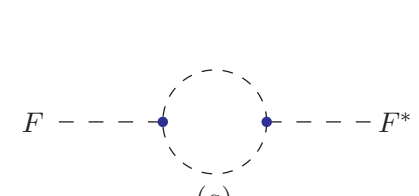

(a)

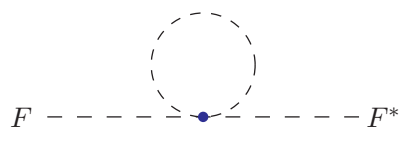

(b)

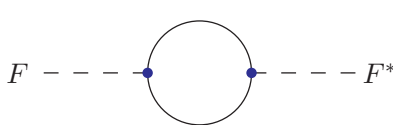

(c)

FIG. 4: Feynman diagrams contributing to the $D$-type soft terms.

where the $\Sigma$ functions are given by

$$
\begin{aligned}
\Sigma_{1}= & \sum_{i} M_{f}\left[\bar{\lambda}_{a}\left(\lambda_{a b} X+m_{a b}\right)^{*}\left(A_{b i} A_{i c}^{\dagger}\right)^{*} \lambda_{c} \frac{-i}{4 \pi^{2}} f\left(m_{1 i}^{2}, M_{f}^{2}\right)+\left(A \rightarrow B, m_{1} \rightarrow m_{2}\right)\right. \\
& \left.+\bar{\lambda}_{a}\left(C_{a i} C_{i b}^{\dagger}\right)^{*}\left(\lambda_{b c} X+m_{b c}\right)^{*} \lambda_{c} \frac{-i}{4 \pi^{2}} f\left(m_{1 i}^{2}, M_{f}^{2}\right)+\left(C \rightarrow D, m_{1} \rightarrow m_{2}\right)\right] \\
\Sigma_{2}= & \sum_{i} M_{F}\left[\bar{\lambda}_{a}\left(\lambda_{a b} X+m_{a b}\right)^{*}\left(A_{b i} C_{i c}^{\dagger}\right)^{*} \bar{\lambda}_{c}^{*} \frac{-i}{4 \pi^{2}} f\left(m_{1 i}^{2}, M_{f}^{2}\right)+\right. \\
& \left.\lambda_{a}^{*}\left(A_{a i} C_{i b}^{\dagger}\right)^{*}\left(\lambda_{b c} X+m_{b c}\right)^{*} \lambda_{c} \frac{-i}{4 \pi^{2}} f\left(m_{1 i}^{2}, M_{f}^{2}\right)+\left(A \rightarrow B, C \rightarrow D, m_{1} \rightarrow m_{2}\right)\right],
\end{aligned}
$$

Repeated indices should be summed over. Those two terms come from the scalar loop (the left plot in Fig. 3), and $\Sigma_{1} \propto M_{f}$ is orders smaller than the second term. That is to say, the holomorphic corrections are dominant by the term proportional to the low energy mass of itself. The fermion loop contributes a terms

$$
\Sigma_{3}=\sum_{i}\left[\lambda_{a} U_{R a i} U_{L i b} \bar{\lambda}_{b} \frac{i}{4 \pi^{2}} M_{2 i} M_{f} f\left(M_{2 i}, M_{f}\right)\right] .
$$

The non-holomorphic terms from Fig. 4 are given by

$$
\begin{aligned}
\Sigma_{4} & =\sum_{i}\left[\bar{\lambda}_{a}\left(\lambda_{a b} X+m_{a b}\right)\left(A_{b i} A_{i c}^{\dagger}\right)^{*}\left(\lambda_{c d} X+m_{c d}\right)^{*} \bar{\lambda}_{d}^{*} \frac{-i}{4 \pi^{2}}\left[\log m_{1 i}^{2}-1\right]+\left(A \rightarrow B, m_{1} \rightarrow m_{2}\right)\right], \\
\Sigma_{5} & =\sum_{i}\left[\bar{\lambda}_{a}^{*} C_{a i} C_{i b}^{\dagger} \bar{\lambda}_{b} \frac{-i}{4 \pi^{2}} m_{1 i}^{2} \log m_{1 i}^{2}+\left(C \rightarrow D, m_{1} \rightarrow m_{2}\right)\right], \\
\Sigma_{6} & =\sum_{i}\left[\bar{\lambda}_{a}^{*} U_{L a i}^{\dagger} U_{L i b} \bar{\lambda}_{b} \frac{i}{4 \pi^{2}} M_{2 i}^{2}\left(2 \log M_{2 i}^{2}-1\right)\right] .
\end{aligned}
$$

The function $f(x, y)$ is defined as

$$
f(x, y)=\frac{x \log x-y \log y}{x-y}-1 .
$$

The messenger fields $(f, \bar{f})$ have similar expressions except for a flavor index. 


\section{B. Soft Mass Terms from the Two-Loop Yukawa Mediation}

The two-loop corrections are dominant when $M=0$ in the hidden sector. Now we calculate such two-loop corrections explicitly via the wave function renormalization method. We use the notation in Ref. [28]. The superpotential is given by

$$
W=\lambda_{a} X \bar{\phi}_{a i} \phi_{a i}+\lambda_{a} \phi_{a i} \bar{F}_{i} f+\bar{\lambda}_{a} \bar{\phi}_{a i} F_{i} \bar{f}+M_{F} F_{i} \bar{F}_{i}+M_{f} f \bar{f} .
$$

In the following calculations only one pair of secondary messenger fields is introduced, thus, $\lambda_{a}=\lambda=\bar{\lambda}_{a}$. According to this method, we have to calculate the threshold effect after integrating out the heavy messengers. The soft terms can be extracted from the light fields' wave-functions that get renormalziation from messengers, which are given by

$$
\begin{aligned}
m_{F F^{*}}^{2} & =-Z_{F}^{\prime \prime} \frac{F^{2}}{M_{h}^{2}}, \\
\left.Z_{F}^{\prime \prime}\right|_{Q=M_{h}} & =\frac{1}{4}\left[\beta_{\lambda}^{(+)} \frac{\partial\left(\Delta \gamma_{F}\right)}{\partial \lambda^{2}}-\Delta \beta_{\lambda} \frac{\partial \gamma_{F}^{(-)}}{\partial \lambda^{2}}\right]_{Q=M_{h}} .
\end{aligned}
$$

The beta-function, anomalous dimension and their change when crossing the messenger scale are given by

$$
\begin{aligned}
\Delta \gamma_{F} & =\frac{-2}{16 \pi^{2}} \lambda^{2}, \quad \gamma_{F}^{(-)}=0, \\
\beta_{\lambda}^{(+)} & =\frac{2 \lambda^{4}}{16 \pi^{2}}(N+2) .
\end{aligned}
$$

With this quantity we obtain

$$
m_{F F^{*}}^{2}=\frac{\lambda^{4}}{\left(16 \pi^{2}\right)^{2}}(N+2) \frac{F^{2}}{M_{h}^{2}} .
$$

Similarly, we have

$$
m_{f f^{*}}^{2}=\frac{\lambda^{4}}{\left(16 \pi^{2}\right)^{2}} N(N+2) \frac{F^{2}}{M_{h}^{2}} .
$$

[1] S. Dimopoulos and H. Georgi, Nucl. Phys. B 193, 150 (1981).

[2] M. Dine, W.Fisler and M. Srednicki, Nucl. Phys. B189, 575 (1981); S. Dimopoulos and S. Raby, Nucl. Phys. B192, 353 (1982); Nucl. Phys. B219, 479 (1983); M. Dine and W.Fisler, Phys. Lett. B110, 227 (1982); Nucl. Phys. B204, 346 (1982); C. Nappi and B. Ovrut, Phys. Lett. B113, 175 (1982); L. Alvarez-Gaume, M. Claudson and M. Wise, Nucl. Phys. B207, 96 (1982); M. Dine and A. E. Nelson, Phys. Rev. D48, 1277 (1993); D51, 1362 (1995); M. Dine, A. Nelson, Y. Nir and Y. Shirman, Phys. Rev. D53, 2658 (1996); 
[3] C. Kolda, Nucl. Phys. Proc. Suppl. 62, 266 (1998); G. F. Giudice and R. Rattazi, Phys. Rept. 322, 419 (1999); S. Dimopoulos, S. Thomas and J. D. Wells, Nucl. Phys. B488, 39 (1997).

[4] E. Poppitz and S. P. Trivedi, Phys. Rev. D 55, 5508 (1997); N. Arkani-Hamed, J. MarchRussell and H. Murayama, Nucl. Phys. B 509, 3 (1998); H. Murayama, Phys. Rev. Lett. 79, 18 (1997); S. Dimopoulos, G. R. Dvali, R. Rattazzi and G. F. Giudice, Nucl. Phys. B 510, 12 (1998);

[5] Z. Komargodski and D. Shih, JHEP 0904, 093 (2009) [arXiv:0902.0030 [hep-th]].

[6] T. T. Dumitrescu, Z. Komargodski, N. Seiberg and D. Shih, JHEP 1005, 096 (2010)

[7] L. Randall, Nucl. Phys. B 495, 37 (1997) [arXiv:hep-ph/9612426].

[8] N. Seiberg, T. Volansky and B. Wecht, JHEP 0811, 004 (2008) [arXiv:0809.4437 [hep-ph]].

[9] M. McGarrie and R. Russo, Phys. Rev. D 82, 035001 (2010) [arXiv:1004.3305 [hep-ph]].

[10] M. Ibe, K. I. Izawa and Y. Nakai, arXiv:0907.2970 [hep-ph].

[11] R. Argurio, M. Bertolini, G. Ferretti and A. Mariotti, JHEP 1003, 008 (2010) [arXiv:0912.0743 [hep-ph]].

[12] K. A. Intriligator, N. Seiberg and D. Shih, JHEP 0604, 021 (2006) [arXiv:hep-th/0602239].

[13] C. Cheung, A. L. Fitzpatrick and D. Shih, JHEP 0807, 054 (2008) [arXiv:0710.3585 [hep-ph]].

[14] D. Shih, JHEP 0802, 091 (2008) [arXiv:hep-th/0703196].

[15] Z. Sun, JHEP 0901, 002 (2009) [arXiv:0810.0477 [hep-th]].

[16] G. F. Giudice and R. Rattazzi, Nucl. Phys. B 511, 25 (1998) [arXiv:hep-ph/9706540].

[17] N. Arkani-Hamed, G. F. Giudice, M. A. Luty and R. Rattazzi, Phys. Rev. D 58, 115005 (1998) [arXiv:hep-ph/9803290].

[18] E. Gorbatov and M. Sudano, JHEP 0810, 066 (2008) [arXiv:0802.0555 [hep-ph]]. K. A. Intriligator and M. Sudano, JHEP 0811, 008 (2008) [arXiv:0807.3942 [hep-ph]]. M. Buican and Z. Komargodski, JHEP 1002, 005 (2010) [arXiv:0909.4824 [hep-ph]].

[19] P. Meade, N. Seiberg and D. Shih, Prog. Theor. Phys. Suppl. 177, 143 (2009) M. Buican, P. Meade, N. Seiberg and D. Shih, JHEP 0903, 016 (2009) L. M. Carpenter, M. Dine, G. Festuccia and J. D. Mason, Phys. Rev. D 79, 035002 (2009)

[20] E. Poppitz and S. P. Trivedi, Phys. Lett. B 401, 38 (1997) [arXiv:hep-ph/9703246].

[21] R. Argurio, M. Bertolini, G. Ferretti and A. Mariotti, arXiv:1006.5465 [hep-ph].

[22] A. Amariti, L. Girardello, A. Mariotti and M. Siani, arXiv:1003.0523 [hep-th].

[23] E. Barnes, K. A. Intriligator, B. Wecht and J. Wright, Nucl. Phys. B 702, 131 (2004) [arXiv:hep-th/0408156].

[24] M. Ibe, Y. Shirman and T. T. Yanagida, arXiv:1009.2818 [hep-ph].

[25] M. McCullough, arXiv:1010.3203 [hep-ph].

[26] A. Giveon, A. Katz, Z. Komargodski and D. Shih, JHEP 0810, 092 (2008) [arXiv:0808.2901 
[hep-th]].

[27] A. Amariti and A. Mariotti, JHEP 0907, 071 (2009) [arXiv:0812.3633 [hep-th]].

[28] A. Delgado, G. F. Giudice and P. Slavich, Phys. Lett. B 653, 424 (2007) [arXiv:0706.3873 [hep-ph]]. 